\title{
Protein and cholesterol's role in the global battle against world cancer epidemic
}

\begin{abstract}
As an ancillary proposition we can note that tumors are more than insular masses of proliferating cancer cells. Instead they are complex tissues composed of multiple distinct cell types that participate in heterotypic interactions with one another. There are the recruited normal cells, which form tumor-associated stroma, as active participants in tumorigenesis rather than passive bystanders. As such, these stromal cells contribute to the development and expressing of certain hallmark capabilities. During the last decades this notion has been solidified and extended, revealing that the biology of tumors can no longer be understood simply by enumerating the traits of the cancer cells but instead must encompass the contributions of the tumor microenvironment to World Cancer Epidemic.
\end{abstract}

Keywords: complex tissues, IBD, epitope, IGPR-1, ECM
Volume 5 Issue I - 2018

\author{
Robert Skopec \\ AXON Researcher, Slovakia
}

Correspondence: Robert Skopec, Dubnik, AXON Researcher, Slovakia,Email zxcbnvm7@gmail.com

Received: October 28, 2017 | Published: January 31, 2018

\section{Introduction}

A role for inflammation in tumorigenesis is now generally accepted, and it has become evident that an inflammatory microenvironment is an essential component of all tumors, including some in which a direct causal relationship with inflammation is not yet proven. Only a minority of all cancers are caused by germline mutations, whereas the vast majority $(90 \%)$ is linked to somatic mutations and environmental factors. Many environmental causes of cancer and risk factors are associated with some form of chronic inflammation. Up to $20 \%$ of cancers are linked to chronic infections, $30 \%$ can be attributed to tobacco smoking and inhaled pollutants, and $35 \%$ can be attributed to dietary factors ( $20 \%$ of cancer burden is linked to obesity). Some type of chronic inflammation that precedes tumor development is caused by immune deregulation and autoimmunity. An example is inflammatory bowel disease (IBD), which greatly increases the risk of colorectal cancer. ${ }^{1}$

Two such attributes are particularly compelling. The first involves major reprogramming of cellular energy metabolism in order to support continuous cell growth and proliferation replacing the metabolic program that operates in most normal tissues and fuels the physiological operations of the associated cells. The second involves active evasion by cancer cells from attack and elimination by immune cells: this capability highlights the dichotomous roles of an immune system that both antagonizes and enhances tumor development and progression. Both of these capabilities may well prove to facilitate the development and progression of many forms of human cancer and therefore can be considered to be emerging hallmarks of cancer. ${ }^{2}$

Most, if not all, solid malignancies trigger an intrinsic inflammatory response that builds up a protumorigenic microenvironment. In addition to cell-autonomous proliferation, certain oncogenes, such as RAS and MYC family members, induce a transcriptional program that leads to remodeling of the tumor microenvironment through recruitments of leucocytes, expression of tumor-promoting chemokines and cytokines, and induction of an angiogenic switch. ${ }^{1,3}$ New findings suggest the management or disruption of the secretion of this protein holds the key to stop cancer from spreading from the pancreas to the liver. See also the role of so called "protein ropes" role in the eating habits, for example in the Alzheimer disease, etc.
This might have a crucial role in the development of bowel cancer, and the bugs that that require antibiotics may induce inflammation, which is known risk for the development of bowel cancer. These findings suggest the potential need to limit the use of antibiotics and sources of inflammation that drive tumor formation. In cancer, proteins are pathologically altered through mutation, they too appear on the cell surface, in this case as neo-epitopes. A cell with a neoepitope probably can be recognized by T-cells, which then destroy the cell. It is known that cancer constantly changes, usually in response to treatment, and will become resistant. It will change its molecular characteristics. There is a need not only for finding those cells and counting them, but actually isolating them down to the single cell level. Researchers want to determine the genomic characteristics of them to figure out what particular gene and protein are expressing, and whether they can use information to determine the best type of treatment.

\section{Proteins may be the key factors of several types of cancer}

Probably it may be a new potential for the treatment if we can study a program targeting special role of proteins in generating cancers. Proteins could be the Evil of Cancer behind the scene, but until now is not generally recognized its destructive function in full manner. It's another goal for future cancer research initiative.

\section{Increased protein in colon cancers}

Colorectal cancer is known as a fourth leading cause of cancerrelated deaths worldwide. In a new study, researchers demonstrate for the first time that a previously uncharacterized protein is increased in colon cancer. This protein is immunoglobulin containing proline rich receptor-1 (IGPR-1) which was recently identified in the same laboratory as a cell adhesion molecule.

The new findings, reported online in journal Oncogenesis, shed light on how IGPR-1 contribute to colon tumor growth and drug resistance. To grow and survive, normal cells needs to attach to extracellular matrix (ECM). Cancerous cells, often bypass this requirement and instead they rely on cell-cell adhesion for survival and growth. By manipulating IGPR-1 expression in colon cancer 
tumor, the researchers revealed that IGPR-1 by promoting tumor cellcell adhesion plays a critical role in colon cancer. The researchers hope to propose a new treatment strategy for patients with colon cancer.

They demonstrate that IGPR-1 by promoting tumor cell-cell adhesion stimulates tumor growth in cell culture and in an experimental model. Blocking IGPR-1 by a specific blocking antibody and shRNA inhibited tumor growth, suggesting a significant therapeutic potential for targeting IGPR-1 in colorectal cancer, as it told corresponding author Nader Rahimi, $\mathrm{PhD}$, associate professor of pathology \& laboratory medicine at Boston University School of Medicine. The researchers also demonstrated that IGPR-1 determines the sensitivity of tumor cells to chemotherapeutic agent, Doxorubicin/Adriamycin and identified the mechanism by which IGPR-1 contributes to drug resistance in colon cancer, a major challenge associated with the treatment of this cancer. ${ }^{4}$

Adhesion to extracellular matrix (ECM) is crucially important for survival of normal epithelial cells as detachment from ECM triggers specific apoptosis known as anoikis. As tumor cells lose the requirement for anchorage to ECM, they rely on cell-cell adhesion multicellular aggregation for survival. Multicellular aggregation of tumor cells also significantly determines the sensitivity of tumor cells to the cytotoxic effects of chemotherapeutics. The expression of immunoglobulin containing and proline-rich receptor-1 (IGPR-1) is upregulated in human primary colon cancer. IGPR-1 promotes tumor multicellular aggregation, and interfering with its adhesive function inhibits multicellular aggregation and, increases cell death.

IGPR-1 supports colon carcinoma tumor xenograft growth in mouse, and inhibiting its activity by shRNA or blocking antibody inhibits tumor growth. IGPR-1 regaulates sensitivity of tumor cells to the chemotherapeutic agent, doxorubicin/adriamycin by a mechanism that involves doxorubicin-induced AKT activation and phosphorilation of IGPR-1 at Ser220. These findings offer novel insight into IGPR-1's role in colorectal cancer target.

\section{Discussion}

It has focused on the role of tumor microenvironment in tumor growth and response to chemotherapeutics. This is particularly relevant to human colorectal cancer, in which, in addition to multiple genetic lesions, other cellular alterations also play roles in the development of carcinoma. IGPR-1 expression is elevated in human primary colon cancers and promotes in vivo and in vitro tumor growth. Interfering with IGPR-1 activity by shRNA or blocking antibody inhibited growth of HCT 116 cells, suggesting that targeting IGPR-1 could offer a novel anti-cancer strategy. IGPR-1 distinctively promotes tumor growth by increasing multicellular aggregation of tumor cells.

IGPR-1 expression in colon tumor cells significantly contributes to the development of resistance of the chemotherapeutic drug, doxorubicin. CRC is the second most common cause of cancer death in men and third in women. The response of CRC patients to current standard-of-care drugs is about $30-40 \%$. Even with the advent of novel targeted anti-cancer drugs, not all patients respond to these agents, epitomizing the fundamental concept of personalized medicine in line with the heterogeneity of CRC. Therefore, further elucidation of IGPR-1 function in CRC could lead to the development of novel therapies that specifically target tumor cell aggregation.
CRC development is a multistep process characterized by numerous genetic and epigenetic lesions. In addition to random genetic lesions, mechanisms by which CRC cells sense their microenvironment (AI!-R.S.) have significant importance in deriving the progression of malignancy and evasion from chemotherapy. As tumor cells lose the requirement for anchorage dependency to ECM for growth and survival, they increasingly rely on multicellular aggregation for survival. It is an evolutionary cellular property that is essential in the embryonic development of organisms. Tumor cells coopt to multicellular aggregation for their survival and, develop resistance to the killing effects of chemotherapeutics. The multicellular aggregation of tumor cells represents a novel target for anti-cancer therapies and adjuvant treatments that aim to enhance CRC chemosensitivity.

IGPR-1 promotes tumor growth both in vivo an in vitro by increasing multicellular aggregation of tumor cells. Loss of adhesion is known to trigger activation of stress-induced pro-apoptotic p38 MAPK. IGPR-1, by increasing multicellular aggregation suppresses of pro-apoptotic p38 activation. The mechanisms by which tumor cells sense their microenvironment institute both respective and permissive roles in tumorigenesis. Cell adhesion molecules (CAMs; cadherins, integrins and immunoglobulin superfamily molecules permit tumor cells to sense their microenvironment, which enables them to resist apoptosis, sustain proliferation, invade and metastasize. IGPR-1 appears to have no effect in the proliferation of cells in 2D cell culture condition, because it promotes cell survival in non-adherent condition, suggesting that IGPR-1 could play a critical role in various stages of tumor metastasis, where tumor cells are not attached to their normal substratum. The acquisition of apoptotic resistance by tumor cells is one of the hallmarks of cancer, and, in many cases, the loss of functional p53 and expression of certain classes of CAMs help tumor cells to avoid cell death and survive. Increased IGPR-1 expression in tumor cells provides a distinct survival mechanism for these cells.

Another important aspect of IGPR-1 expression in colon cancer cells is its ability to modify the response of tumor cells to the chemotherapeutic effects of doxorubicin. IGPR-1 was uniquely phosphorylated at Ser200 in response to doxorubicin, in an AKTdependent manner. Identification of Ser220 phosphorylation on IGPR1 has significant implications for its use as a potential biomarker in response to treatment and tumor progression. The mechanisms by which tumor cells sense their microenvironment to impede apoptosis associated with the loss of adhesion to substratum and response to the killing effects of chemotherapeutics. Molecular mechanism is based on fact that IGPR-1 promotes tumor cell survival by multicellular aggregation. It was also established the functional importance of IGPR-1 in CRC tumor cells in response to chemotherapeutics. IGPR-1 provides a unique ability to evade apoptosis, grow without anchoring to ECM, and develop resistance to conventional chemotherapies. Targeted therapies directed toward blocking tumor cells' ability to sense their microenvironment, a mechanism that allows tumor cells to survive through multicellular aggregation, offers an attractive novel anti-cancer therapeutic approach

\section{Immune cells promote cancer}

Researchers at the Children's Center and Blood Diseases at Children's Hospital Los Angeles (CHLA) have identified new findings about an immune cell called a tumor-associated macrophage (TAM) that promotes cancer instead of fighting it. They have identified the molecular pathway, known as STAT3, as the mechanism the immune cell uses to foster neuroblastoma, a pediatric cancer. They have 
also demonstrated use of a clinically available agent, ruxolitinib, to block the pathway. Results of the study were published in the journal Oncotarget on September 20, 2017.

Neuroblastoma is the second most common solid tumor effecting children. Individuals with high-risk disease have a mortality rate of approximately $50 \%$. Certain conditions are associated with high-risk disease. High levels of some chemicals involved with inflammation and the presence of an immune cell called a tumor-associated macrophage (TAM) are associated with high-risk disease and lower survival rates. Macrophages are a type of immune cell that typically function to battle disease, not encourage it. The macrophages are essentially co-opted by the tumor cells to help them grow, said Ashgharzadeh, ${ }^{5}$ director of the basic and Translational Neuroblastoma program at CHLA and lead investigator of the study. They're trying to find out more about the mechanisms that enable TAMs to help cancer grow so that they can target the pathways they use and block their pro-tumor effect.

The team wanted to find out whether effective therapeutic approaches for children with neuroblastoma could be based on targeting inflammation-associated biologic pathways in the area surrounding the tumor the tumor microenvironment. Using a mouse model to examine the activity of TAMs within the tumor microenvironment, the researchers observed the recruitment and polarization of TAMs which enhance the ability of neuroblastoma to spread and grow. They found that TAMs exhibit a dual role - not only nourishing the neuroblastoma, but also effectively helping them to evade the good immune cells seeking to kill the tumor cells. To study the effect of TAMs on neuroblastoma cell growth and proliferation, the investigators co-cultured both mouse and human neuroblastoma cells with TAMs and found a significant increase compared to tumor cells without TAMs.

In an effort to find out what the TAMs were secreting that caused stimulation of tumor cells, the researchers targeted IL-6, an immune substance known to cause proliferation of certain types of cancer. Using a mouse model that lacked IL-6, they still observed increased tumor growth. In these experiments, they noted activation of the STAT3 cell-signaling pathway known to promote tumor growth preceding an increase of MYC a gene that drives many types of cancer. These findings led them to target the STAT3 pathway. Using a clinically available drug, ruxolitinib, known to block the STAT3 pathway, the researchers co-cultured both human and mouse TAMs and neuroblastoma cells. They observed that the immune cells no longer supported tumor growth.

Targeting STAT3 may be promising approach to block interactions between tumor cells and the traitorous immune cells, and a way to improve outcomes for children with high-risk neuroblastoma, said Ashgharzadeh, ${ }^{5}$ who is also a professor of pediatrics with the Keck School of Medicine of the University of Southern California. According to him, the next step is to combine agents that block the STAT3 pathway with drugs that have been effective in treating neuroblastoma. ${ }^{5}$

\section{Controversial, inflammation \\ two-faced therapy-induced}

Even dominant oncogenes such as v-Scr or K-Ras are unable to induce cancer in adult animals unless accompanied by injury and subsequent tissue regeneration. Also a strong tumor-associated inflammatory response can be initiated by cancer therapy. Radiation and chemotherapy cause massive necrotic death of cancer cells and surrounding tissues, which in turn triggers an inflammatory reaction analogous to a wound-healing response. The net outcome of therapyinduced inflammation is controversial (two-faced), as on the one hand it can have tumor-promoting functions just like the necrosis that accompanies rapid tumor growth. On the other hand it can enhance the cross-presentation of tumor antigens and subsequent induction of an antitumor immune response.

However, it is safe to assume that tumor-promoting inflammation and antitumor immunity coexist at different points along path of tumor progression and that environmental and microenvironmental conditions dictate the balance between the two. ${ }^{1}$ The most frequently found immune cells within the tumor microenvironment are tumorassociated macrophages (TAMs) and T cells. TAMs mostly promote tumor growth and may be obligatory for angiogenesis, invasion, and metastasis. High TAM content generally correlates with poor prognosis.

TAMs are one of the most important players in the inflammations and cancer area and an important source of cytokines. ${ }^{4}$ Macrophages can be classified into M1 and M2 types. M1 macrophages, activated by IFN $\gamma$ and microbial products, express high levels of proinflammatory cytokines (TNF- $\alpha$, IL-1, IL-6, IL-12, or IL-23), major hisocompatibility complex (MHC) molecules, and inducible nitric oxide synthase and are capable of killing pathogens and priming antitumor immune responses. By contrast, M2 or alternatively activated macrophages, which are induced in vitro by IL-4, IL-10, and IL-13, down regulate MHC class II and IL-12 expression and show increased expression of the anti-inflammatory cytokine IL-10, scavenger receptor A, and arginase. Most TAMs are considered to have an M2 phenotype while promoting tumor angiogenesis and tissue remodeling.

However, most confirmed tumor-promoting cytokines are M1 cytokines, whereas IL-10, and M2 cytokine, may be tumor-suppressive as shown in colorectal cancer. Macrophages M1 and M2 are plastic and their phenotype is defined by their gene expression profile rathet than by deterministic differentiation pathways and lineage choices. Tumor initiation is a process in which normal cells acquire the fist mutational hit that sends them on the tumorigenic track by providing growth and survival advantages over their neighbors.

\section{Zinc selectively halts growth of cancer cells}

Zinc supplements can significantly inhibit the proliferation of esophageal cancer cells, according to a new study co-authored by a University of Texas at Arlington (UTA) researcher Associate Professor of Nursing and Health Innovation Zui Pan. Previous studies had shown that zinc is essential for maintaining human health and protects the esophagus from cancer. It has never been fully understood why zinc has the ability to prevent cancer in the esophagus. In this study, a team discovered that zinc selectively halts the growth of cancer cells, but not normal esophageal epithelial cells. The finding was published in September issue of The FASEB Journal, the official journal of the Federation of American Societies for Experimental Biology.

Esophageal cancer is the sixth leading cause of human World Cancer Epidemic, according to the National Cancer Institute. The institute estimates that there were almost 16,000 esophageal cancer deaths in the United States in 2016. The average five-year survival rate is less than $20 \%$. Professor Pan said this study could provide a pathway for better esophageal prevention and treatment. Zinc 
deficiency has been found in many cancer patients. Both clinical data and animal studies have shown that this mineral is very important for overall body health and for cancer prevention. Yinc is an important element in many proteins and many enzymes. Therefore the absence of zinc makes it impossible for cells to function.

Previously researchers didn't know why the same physiological concentrations of zinc inhibit cancer cell growth but not normal cells. Above study, for the first time to our knowledge, reveals that zinc impedes overactive calcium signals in cancer cells, which is absent in normal cells, and thus zinc selectively inhibits cancer cell growth. It now appears that zinc and calcium have a cross talk, meaning that they can be linked. An insufficient amount of zinc can lead to the development of cancers and other diseases. That's why it is important to have a good diet, Pan said. Zinc enriched foods include spinach, flax seeds, beef, pumpkin seeds and seafood like shrimps and oysters.

Pan said that in the future they will study these two signals link, how they impact each other and how researchers can take advantageof what they know. Such a step will guide them in developing a better prevention and treatment strategy. Annie Bavier, dean of UTA's College of Nursing and Health Innovation, called Pan's study a classic example of UTA's commitment to high impact research. It re-affirms UTA's position as a major player in the global battle against World Cancer Epidemic. Pan's work on esophageal cancer gets straight to the heart of goal at the College of Nursing and health Innovation to help solve health problems to build healthier world. ${ }^{6}$

\section{Cancer-eating machines}

A team of researchers has engineered smart protein molecules that can reprogram white blood cells to ignore a self-defense signaling mechanism that cancer cells use to survive and spread in the body. The advance could lead to a new method of re-engineering immune cells to fight cancer and infectious diseases. The team successfully tested this method in a live cell culture system. The work was led by bioengineering professors Peter Yingxiao Wang and Shu Chien with collaborating professors Victor Nizet and Xiaodong Xu, all at the University of California San Diego, along with researchers from the University of Illionis at Urbana-Champaign. The team published their work in September issue of nature Communications.

The smart proteins, called iSNAPs (integrated sensing and activating proteins), are designed to detect precise molecular signals in live cells and in response, act upon those signals to enable the cells to fight disease or perform other beneficial functions. This study is the first to demonstrate how both sensing and activating capabilities can be combined into a single molecule. The researchers inserted their iSNAPs into a type of white blood cells called macrophages and demonstrated that they dramatically enhanced the macrophages ability to engulf and destroy rapidly dividing cancer cells.

Macrophages are white blood cells that play a significant role in the immune system. Part of their task is to remove foreign particles and harmful organisms such as pathogens and cancer cells by digesting them. When a macrophage binds to a cancer cell or other foreign invader, surface proteins on the macrophage called Fc gamma receptors send out an eat me signal that prompts the macrophage to engulf and destroy the invader. However, cancer cells have a special protection mechanism that contributes to their deadly disease potential. They have a surface protein called CD47 that interacts with the macrophage's SIRP- $\alpha$ surface protein to send out a negating don't eat me signal.
The secret to getting the iSNAP technology to work involves reconfiguring the battleground between the cancer cells and the immune system. The iSNAPs essentially rewire macrophages to override this don't eat me signal and interpret it as an eat me signal. The iSNAPs have a sensing component that detects the key molecular event that occurs inside a macrophages when its SIRP- $\alpha$ surface protein interacts with CD47 on the cancer cell. In response, the iSNAPs have an activating component that immediately transforms to produce a green/yellow light signal, giving researchers a way to visualize the molecular activity through a microscope. The activating component is also triggered to release an enzyme that initiates a cascade of events that enables the macrophage to engulf the cancer cell. What's noteworthy is that this response time is very fast it happens within seconds to minutes.

Wang noted that the iSNAPs design could in principle be modified for other uses, such as re-ingeneering immune cells to kill bacteria. They could also be applied to other immune cell types such as T cells for multi-pronged cancer therapy. Moving forward, the team is planning to test the iSNAPs in mice to see how they could perform in vivo. The researchers are also interested in studying whether the iSNAPs can be harnessed to enhance other native cellular functions to correct various disease pathologies. ${ }^{7}$

\section{New strategy for halting brain tumors}

There are very few specific or targeted inhibitors that are used in the treatment of brain cancer. There's really a dire need for new therapies and new ideas. MIT biologists have discovered a fundamental mechanism that helps brain tumors called glioblastomas grow aggressively. After blocking this mechanism in mice, the researchers were able to halt tumor growth. The researcher also identified a genetic marker that could be used to predict which patients would most likely benefit from this type of treatment. Glioblastoma is usually treated with radiation and the chemotherapy drug temozolamide, which may extend patients lifespan but in most cases do not offer a cure. The reason is that there are very specific or targeted inhibitors that are used in the treatment of brain cancer. Recently there's a big dire request for new therapies and new ideas in this field, says Michael Hemman, an associate professor of biology at MIT, a member of MIT's Koch Institute for Integrative Cancer Research.

Drugs that block a key protein involved in the newly discovered process already exist, and at least one is in clinical trials to treat cancer. However, most of these inhibitors do not cross the blood-brain barrier, which separates the brain from circulating blood and prevents large molecules from entering the brain. The MIT team hopes to develop drugs that can cross this barrier, possibly by packaging them into nanoparticles. The study, which appears in Cancer Cell on Sept. 28, is a collaboration between the labs of Michael Hemman, an associate professor of biology at MIT, a member of MIT's Koch Institute for Integrative Cancer Research, Jacqueline Lees, associate director of the Koch Institute and the Virginia and D.K. Ludwig Professor for cancer research, and Philip Sharp, an MIT Institute Professor and member of the Koch Institute. The paper's lead authors are former MIT postdoc Christian Braun, recent PhD recipient Monica Stanciu, and research scientist Poul Boutz.

Several years ago, M. Stanciu and Ch. Braun came up with the idea to use a type of screen known as shRNA to seek genes involved in glioblastoma. This test involves using short strands of RNA to block the expression of specific genes. Using this approach, researchers can 
turn off thousands of different genes, one per tumor cell, and then measure the effects on cell survival. One of the top hits from this screen was the gene for a protein called PRMT5. When this gene was turned off, tumor cell stopped growing. Previous studies had linked high levels of PRMT5 to cancer, but the protein is an enzyme that can act on hundreds of other proteins, so researchers weren't sure exactly how it was stimulating cancer cell growth.

Further experiments in which they analyzed other genes affected when PRMT5 was inhibited led them to hypothesize that PRMT5 was using a special type of gene splicing to stimulate tumor growth. Gene splicing is required to snip out portions of messenger RNA known as introns, that are not needed after the gene is copied into mRNA. In 2015, Boutz and others in Sharp's lab discovered that about 10$15 \%$ of human mRNA strands still have 1-3 detained introns, even though they are otherwise mature. Because of those introns, these mRNA molecules can't leave the nucleus. These strands are basically a mRNA reservoir. These unproductive isoforms are sitting in the nucleus, and only thing that keeps them from being translated is one intron, says Ch. Braun, who is now a physician-scientist at Ludwig Maximillian University of Munich.

In the new study, the researchers discovered that PRMT5 plays a key role in regulating this type of splicing. They speculate that neural stem cells utilize high levels of PRMT5 to guarantee efficient splicing and therefore expression of proliferation genes. As the cells move toward their mature state, PRMT5 levels drop, detained intron levels rise, and those messenger RNA associated with proliferation get stuck in the nucleus. When brain cells become cancerous, PRMT5 are typically boosted and the splicing of proliferation-associated RNA is improved, ultimately helping the cells to grow uncontrollably.

When the researchers blocked PRMT5 in tumor cells, they found that the cells stopped dividing and entered a dormant, nondividing state. PRMT5 inhibitors also halted growth of glioblastoma tumors implanted under the skin of mice, but they did not work as well in tumors located in the brain, because of the difficulties in crossing the blood-brain barrier. Unlike many existing cancer treatments, the PRMT5 inhibitors did not appear to cause major side effects. The researchers believe this may be because mature cells are not as dependent as cancer cells on PRMT5 function.

PRMT5 has a lot of roles, and until now, it has not been clear what is the pathway that is really important for its contributions to cancer. What they have found is that one of the key contributions is in this RNA splicing mechanism, and furthermore, when RNA splicing is disrupted, that key pathway is disabled. The researchers also discovered a biomarker that could help identify patients who would be most likely to benefit from a PRMT5 inhibitor. This marker is a ratio of two proteins that act as co-factors for PRMT5's activity, and reveals whether PRMT5 in those tumor cells is involved in splicing or some other cell function.

The MIT team is now looking into the potential role of PRMT5 in other types of cancer, including lung tumors. They also hope to identify other genes and proteins involved in the splicing process they discovered, which could also make good drug targets. It really is an amazing example of the creativity that young researchers at MIT have. They are fearless. ${ }^{8}$

\section{Probiotic bacteria change intestinal flora in colon cancer}

Living lactic acid bacteria, probiotics, can change the intestinal microflora of patients with cancer of the colon. These are the findingsa of a study published in the journal BMJ Open Gastroenterology. The probiotic strains used in this study are promising positive factor for the continued development of treatments for colon cancer, confirms Yvonne Wettergren, Associate professor in Molecular Medicine at the Institute of Clinical Sciences, Sahlgrenska Academy. The study shows that the intestinal microflora composition, studied in biopsies, was different in tumor tissue and the surrounding mucosa in patients with colon cancer compared with healthy individuals.

When the microflora among those with cancer was modified by treatment with probiotics in tablet form, the amount of bacteria that produce butyric acid increased. This acid has documented antiinflammatory effects on the colon and also inhibits the growth of cancer cells in the intestines. A component of the probiotic strains used in the study, lactobacillus acidophilus NCFM, previously proved to reduce the growth of colon tumors in mice, and also reduced the amount of carcinogenic decomposition products, so-called metabolites, in the intestines of humans.

Through yet another component, Bifidobacterium lactis B1-04, the preparation was given anti-inflammatory properties. The actual tablets were designed so that the bacteria would really be exposed in the colon, which is required for the clinical documentation. The study builds on the principle that the human intestinal microflora affects the metabolism, interacts with the immune system and protects against pathogens, toxins and other factors that cause World cancer Epidemic. It thereby has the potential to have a strong impact on general health and well-being.

What can be viewed as a healthy microflora can vary significantly from person to person, but more recent studies have shown that the intestinal flora often deviates from its normal stable state in diseases of the gastrointestinal tract, as well as the development of the metabolic syndrome. According to the current study, the microbiological imbalance seen in patients with colon cancer changes with the help of probiotic bacteria, given that they are dosed and administered in the way done in the study.

The study comprises a total of 15 patients who already had malignant cancer, and who were followed until operation. The researchers are now going to continue with a larger study of patients who have premalignant stages of cancer, intestinal polyps. They hope to be able to work with prevention in this area.

\section{Calcium-binding protein inhibits cholesterol efflux}

The research began with studying the role of a calciumbinding protein. They noticed an extreme rise of blood cholesterol concentration in mice when the protein was not present! To follow up on this observation, Professor Marek Michalak with colleagues discovered the physiological link between calcium and cholesterol is also preserved in worms.

The CARC-cholesterol interaction is of high affinity, lipid-specific, concentration-dependent, and sensitive to single-point mutations. The CARC motif is generally located in the outer membrane leaflet 
and its reverse sequence CRAC in the inner one. Their simultaneous presence within the same transmembrane domain obeys a "mirror code" controlling protein-cholesterol interactions in the outer and inner membrane leaflets. ${ }^{9}$ Deciphering this code enabled us to elaborate guidelines for the detection of cholesterol-binding motifs in any membrane protein. ${ }^{10}$

It is a first comprehensive report of patient-derived circulating tumor cells at the gene expression level, so we now have a clearer picture of the signaling pathways that allows them to establish brain metastases. By comparing the whole genome expression patterns of CTCs isolated from patient blood samples diagnosed with or without $\mathrm{BCBM}$, they uncovered a 126 gene-signature that is specific to these brain metastatic CTCs, said Debasish Boral, Ph.D., the paper's first author and a research associate with the Biomarker Research Program at Houston Methodist Research Institute.

The $\mathrm{ABC}$ transporter ABCA1 has been implicated to control cholesterol efflux in a variety of cell types including macrophages, fibroblasts, and intestinal epithelial cells. Anionic peptide fraction/ calcium-binding protein (APF/CBP) inhibitedApoA-1 mediated cholesterol efflux from normal fibroblasts in a dose dependent manner but had no effect on specific efflux to methyl-beta cyclodextrin or phosphatidylcholine liposomes. In ABCA1 deficient fibroblasts no effect of APF/CBP on efflux was seen. We conclude that APF/CBP specifically interferes with ApoA-I mediated cholesterol trafficking. Probably the competitive binding to ABCA1 may explain the decreased ApoA-I mediated efflux from fibroblasts.

Cholesterol controls the activity of a wide range of membrane receptors through specific interactions and identifying cholesterol recognition motifs is therefore critical for understanding signaling receptor function. The membrane-spanning domains of the paradigm neurotransmitter receptor for acetylcholine (AChR) display a series of cholesterol consensus domains (CARC). The CARC-cholesterol interaction is of high affinity, lipid-specific, concentration-dependent, and sensitive to single-point mutations. The CARC motif is generally located in the outer membrane leaflet and its reverse sequence CRAC in the inner one. Their simultaneous presence within the same transmembrane domain obeys a "mirror code" controlling proteincholesterol interactions in the outer and inner membrane leaflets Deciphering this code enabled us to elaborate guidelines for the detection of cholesterol-binding motifs in any membrane protein. ${ }^{10}$

\section{Cholesterol cut-off switch for engine of cancer-killing cells}

Researchers have just discovered how the engine that powers cancer-killing cells functions. This research also highlights how that engine is fuelled and that cholesterol-like molecules, called oxysterols, act as a cut-off switch making it hard for the Natural Killer cells to win the war against cancer. The scientists, led by Ussher Assistant Professor in Immunometabolism at Trinity College Dublin, Dr. David Finlay, have just published their findings in leading journal Nature Immunology. They report a previously unknown metabolic switch, which is essential for initiating the anti-tumor actions of Natural Killer cells.

Natural Killer (NK) cells as immune cells play an important role in our defence against cancer, as they can directly kill tunor cells. Once activated, NK cells increase their uptake of cellular fuel, which is converted into energy by a biochemical engine. These engines and their fuel thus power the all-important tumor-killing machinery of the
NK cells. The new research shows that activated NK cells use a very different engine configuration to that observed in other immune cells and the key factor that switches NK cells to this engine configuration is a protein called Srebp. When the researchers used oxysterols to prevent this switch from occurring, the NK cells failed to kill tumor cells.

The function of Srebp the key factor that controls the energy production in NK cells is known to be blocked by cholesterol and cholesterol-like molecules called oxysterols. Their findings reveal a previously unknown way by which the cancer-killing functions of NK cells can be disrupted. As tumor cells produce oxysterols and cholesterol levels tend to be higher in patients with obesity, the researchers believe they may now have part of the explanation for why NK cells typically perform poorly in patients living with cancer and obesity. The next step is to investigate whether the functions of NK cells are indeed impaired in individuals with high cholesterol level, and whether cholesterol lowering interventions can restore NK cell function in these patients, said Finlay. ${ }^{11}$

\section{Acknowledgements}

The author gratefully acknowledge the assistance of Dr.Marta Ballova, Ing. Konrad Balla, Livuska Ballova, and Ing. Jozef Balla.

\section{Conflict of interest}

The author declares no conflict of interest.

\section{References}

1. Grivennikov SI, Greten FR, Karin M. Immunity, Inflammation, and Cancer. Cell. 2010;140(6):883-899.

2. Hanahan D, Weinberg RA. Hallmarks of cancer: the next generation. Cell. 2011;144(5):646-674.

3. Colotta F, Allavena P, Sica A, et al. Cancer-related inflammation, the seventh hallmark of cancer: links to genetic instability. Carcinogenesis. 2009;30(7):1073-1081.

4. Woolf N, Pearson BE, Bondzie PA, et al. Targeting tumor multicellular aggregation through IGPR-1 inhibits colon cancer growth and improves chemotherapy. Oncogenesis. 2017;6(9):e378.

5. Ashgharzadeh S. Investigators identify pathway that causes immune cells to support cancer, instead of killing it. When Good Immune Cells Turn Bad, USA; 2017

6. Choi S, Cui C, Luo Y, et al. Selective inhibitory effects if zinc on cell proliferation in esophageal squamos cell carcinoma through Orai1. FASEB J. 2017;32(1):404-416.

7. Sun J, Lei L, Tsai CM, et al. Engineered proteins with sensing and activating modules for automated reprogramming of cellular functions. Nat Commun. 2017;8(1):477.

8. Braun CJ, Stanciu M, Boutz PL, et al. Coordinated splicing of regulatory detained introns within oncogenic transcripts creates an exploitable vulnerability in malignant glioma. Cancer Cell. 2017;32(4):411-426.

9. https://www.news-medical.net/news/20170921/Researchers-identifymolecular-pathway-that-causes-immune-cell-to-promote-cancer.aspx

10. Fantini J, Di Scala C, Evans LS, et al. A mirror code for protein-cholesterol interactions in the two leaflets of biological membranes. Sci Rep. 2016;6:21907.

11. Finlay D. Scientists discover the engine that powers cancer-killing NK cells. Trinity News and Events, UK; 2017. 